\title{
Measurement report: Dual-carbon isotopic characterization of carbonaceous aerosol in Beijing and Xi'an: distinctions in primary versus secondary sources
}

Haiyan Ni ${ }^{1,2}$, Ru-Jin Huang ${ }^{1,3}$, Max M. Cosijn ${ }^{2}$, Lu Yang ${ }^{1}$, Jie Guo ${ }^{1}$, Junji Cao ${ }^{1,3}$, and Ulrike Dusek $^{2}$

${ }^{1}$ State Key Laboratory of Loess and Quaternary Geology, Center for Excellence in Quaternary Science and Global Change, Key Laboratory of Aerosol Chemistry and Physics, Institute of Earth Environment, Chinese Academy of Sciences, Xi'an, 710061, China

${ }^{2}$ Centre for Isotope Research (CIO), Energy and Sustainability Research Institute Groningen (ESRIG), University of Groningen, Groningen, 9747 AG, the Netherlands

${ }^{3}$ Institute of Global Environmental Change, Xi'an Jiaotong University, Xi'an, 710049, China

Correspondence: Ru-Jin Huang (rujin.huang@ieecas.cn) 


\section{S1. Estimation of $r_{\mathrm{bb}}$ and $\boldsymbol{r}_{\text {fossil }}$}

$r_{\mathrm{bb}}$ and $r_{\text {fossil }}$ are primary OC/EC ratio for biomass burning and fossil fuel combustion, respectively. $r_{\text {fossil }}$ is the weighted average of (POC/EC) $)_{\text {coal }}$ (i.e., $\left.r_{\text {coal }}\right)$, and (POC/EC) $)_{\text {vehicle }}\left(r_{\text {vehicle }}\right)$. The weight associated with $r_{\text {coal }}$ (denoted as $w_{\text {coal }}$ ) is the relative contribution of coal combustion to fossil EC. That is,

$$
w_{\text {coal }}=\frac{\mathrm{EC}_{\text {coal }}}{\mathrm{EC}_{\text {fossil }}}=\frac{\mathrm{EC}_{\text {coal }}}{\mathrm{EC}_{\text {coal }}+\mathrm{EC}_{\text {liq.fossil }}}
$$

where $\mathrm{EC}_{\text {fossil }}$ is the sum of $\mathrm{EC}_{\text {coal }}$ and $\mathrm{EC}$ from liquid fossil fuel combustion (i.e., vehicle emissions; $\mathrm{EC}_{\text {liq.fossil). }}$

Eq. (S1) can be formulated as:

$$
w_{\text {coal }}=\frac{f_{\text {coal }}}{f_{\text {fossil }}}=\frac{f_{\text {coal }}}{f_{\text {coal }}+f_{\text {liq.fossil }}}
$$

where $f_{\text {coal }}$ and $f_{\text {liq.fossil }}$ are the relative contribution of coal combustion and liquid fossil fuel combustion to EC, respectively. The sum of $f_{\text {coal }}$ and $f_{\text {liq.fossil }}$ is $f_{\text {fossil }}$ of $\mathrm{EC}$, which is well constrained by $\mathrm{F}^{14} \mathrm{C}$ of EC. The PDFs of $f_{\text {coal }}$ and $f_{\text {liq.fossil }}$ (e.g., Fig. S4), derived from the Bayesian calculations detailed in Sect. 2.4 of the main text, are used to calculated the PDFs of $w_{\text {coal }}$.

Best estimates of $r_{\mathrm{bb}}(4 \pm 1$; average $\pm \mathrm{SD}), r_{\text {coal }}(2.38 \pm 0.44)$, and $r_{\text {vehicle }}(0.85 \pm 0.16)$ are defined through a literature search as described in $\mathrm{Ni}$ et al. (2018), and their values are comparable to values used in earlier studies (Zhang et al., 2014, 2015).

\section{S2. Uncertainties of ${ }^{14} \mathrm{C}$ source apportionment results}

To propagate uncertainties, we conducted a Monte Carlo simulation with 10000 individual calculations to propagate experimental uncertainties and uncertainties in parameters (e.g., $\mathrm{F}^{14} \mathrm{C}_{\mathrm{nf}}$, $r_{\mathrm{bb}}$ and $\left.r_{\text {fossil }}\right)$ following Eq. (3-12).

For each calculation, $\mathrm{F}^{14} \mathrm{C}$ and mass of $\mathrm{EC}$ and $\mathrm{OC}$ were randomly chosen from a normal distribution symmetric around the measured values with the experimental uncertainties as standard deviation (SD). For $\mathrm{F}^{14} \mathrm{C}_{\mathrm{nf}}, r_{\mathrm{bb}}, r_{\text {coal }}$ and $r_{\text {vehicle, }}$, random values of each parameter were chosen from a triangular frequency distribution with its maximum frequency at the central value and 0 frequency at the lower limit and upper limit of each parameter. For $w_{\text {coal }}$, random values from the respective probability density function (PDF) of $w_{\text {coal }}$ were used (Supplement S1). In this way 10000 random sets of variables were generated. For $f_{\mathrm{bb}}(\mathrm{EC}), f_{\mathrm{nf}}(\mathrm{OC}), \mathrm{EC}_{\mathrm{bb}}, \mathrm{EC}_{\text {fossil }}, \mathrm{OC}_{\mathrm{nf}}$ and $\mathrm{OC}_{\text {fossil }}$, the derived mean represents the best estimate, and the SD represents the combined uncertainties (Table S3). For $\mathrm{POC}_{\mathrm{bb}}, \mathrm{OC}_{\mathrm{o}, \mathrm{nf}}, \mathrm{POC}_{\text {fossil }}, \mathrm{SOC}_{\text {fossil }}, \mathrm{SOC}$ and $f_{\text {fossil }}(\mathrm{SOC})$, the median value is considered as the best estimate and the interquartile ranges (25th-75th percentile) represent the combined uncertainties, because the PDFs of $\mathrm{POC}_{\text {fossil }}$ and $\mathrm{SOC}_{\text {fossil }}$ are asymmetric (Fig. S5, Table S4). The median values for $\mathrm{POC}_{\mathrm{bb}}$ and $\mathrm{OC}_{\mathrm{o}, \mathrm{nf}}$ are very close to their mean values due to their symmetric PDFs (Fig. S5). 

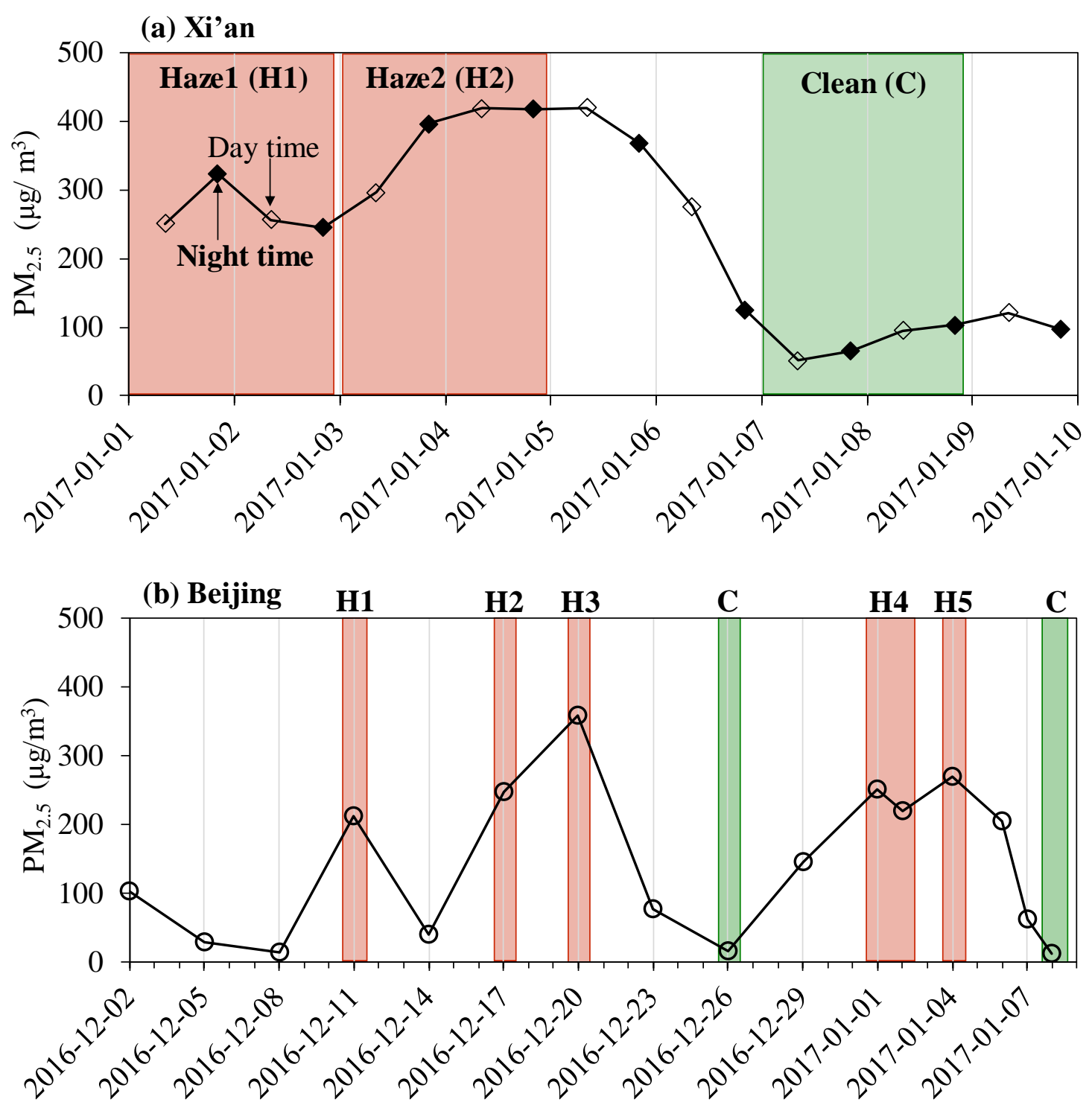

Figure S1. $\mathrm{PM}_{2.5}$ mass concentrations $\left(\mu \mathrm{g} \mathrm{m}^{-3}\right)$ in Xi'an and Beijing during the measurement periods. The dashed areas indicate the selected samples for ${ }^{14} \mathrm{C}$ analysis. (a) $12 \mathrm{~h}$ averaged $\mathrm{PM}_{2.5}$ concentrations in Xi'an, the open symbols represent daytime samples, the filled square symbols represent nighttime samples. (b) $24 \mathrm{~h}$ averaged $\mathrm{PM}_{2.5}$ concentrations in Beijing. Samples selected for ${ }^{14} \mathrm{C}$ analysis are highlighted in red (indicating haze periods) and green (clean periods). For details, see Table S1. 


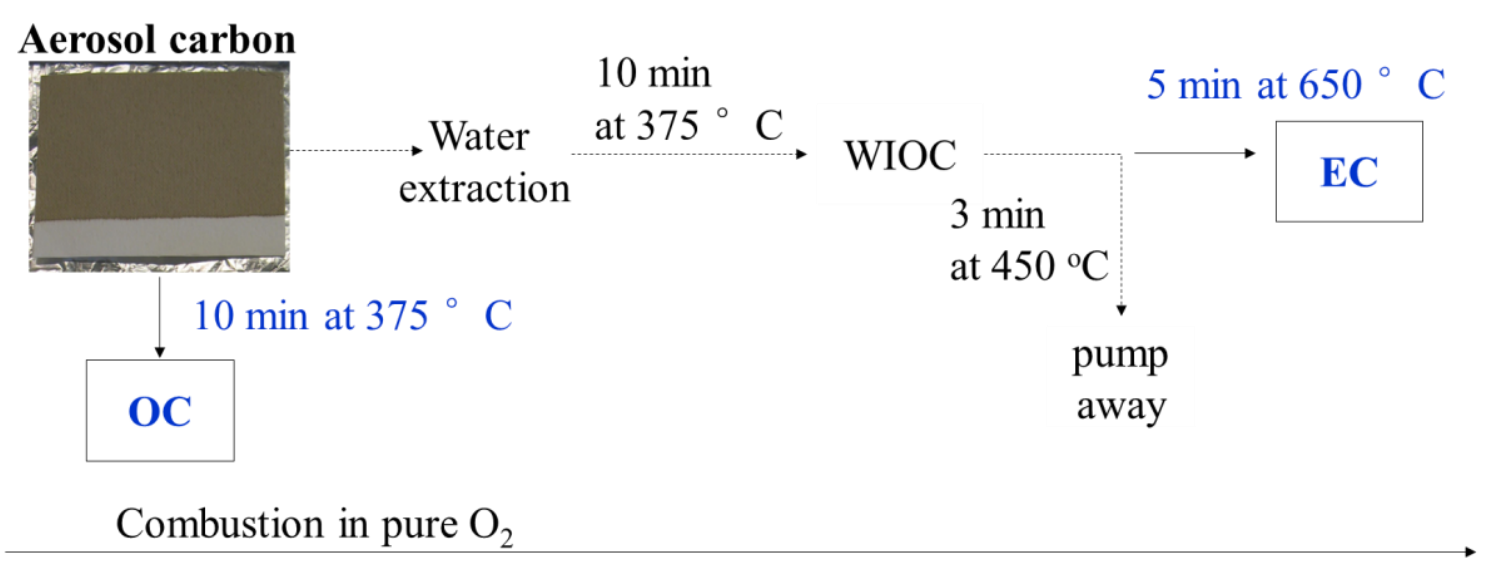

Figure S2. Temperature protocol of $\mathrm{OC}$ and $\mathrm{EC}$ isolation for ${ }^{14} \mathrm{C}$ measurements was implemented on aerosol combustion system (Dusek et al., 2014) in pure $\mathrm{O}_{2}$. OC is extracted by combusting the filter samples at $375^{\circ} \mathrm{C}$ for $10 \mathrm{~min}$. To isolate EC, OC is completely removed by 3 steps in order: water-extraction of filter samples (i.e., removal of watersoluble OC), combustion at $375{ }^{\circ} \mathrm{C}$ for $10 \mathrm{~min}$ (removal of water-insoluble OC; WIOC) in $\mathrm{O}_{2}$, and combustion at $450{ }^{\circ} \mathrm{C}$ for $3 \mathrm{~min}$ in $\mathrm{O}_{2}$ (removal of the most refractory OC). Then, EC is isolated by heating the remaining carbon at $650{ }^{\circ} \mathrm{C}$ for $5 \mathrm{~min}$ in $\mathrm{O}_{2}$. Details can be found in Zenker et al. (2017) and Ni et al. (2018). 


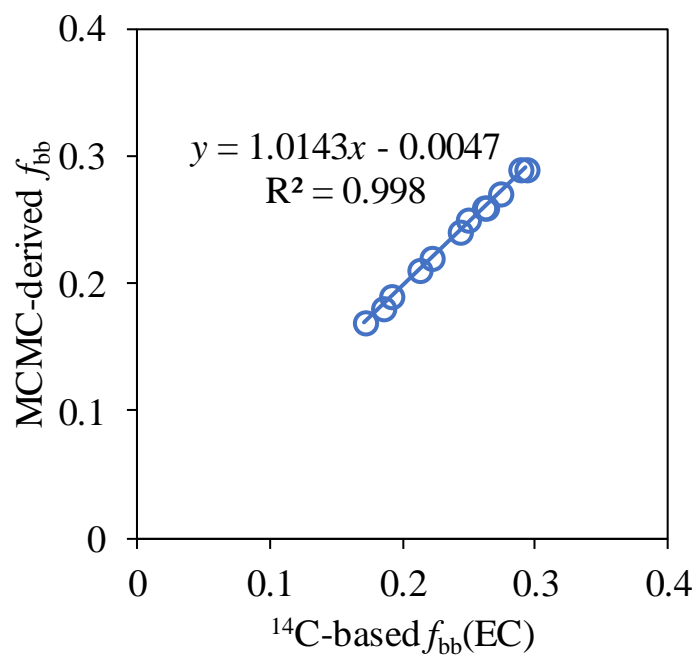

Figure S3. Comparison between the MCMC-derived fraction of biomass burning EC ( $f_{\mathrm{bb}}$ derived from MCMC; median) and that obtained from radiocarbon data $\left({ }^{14} \mathrm{C}-\right.$ based $f_{\mathrm{bb}}(\mathrm{EC})$; mean). 

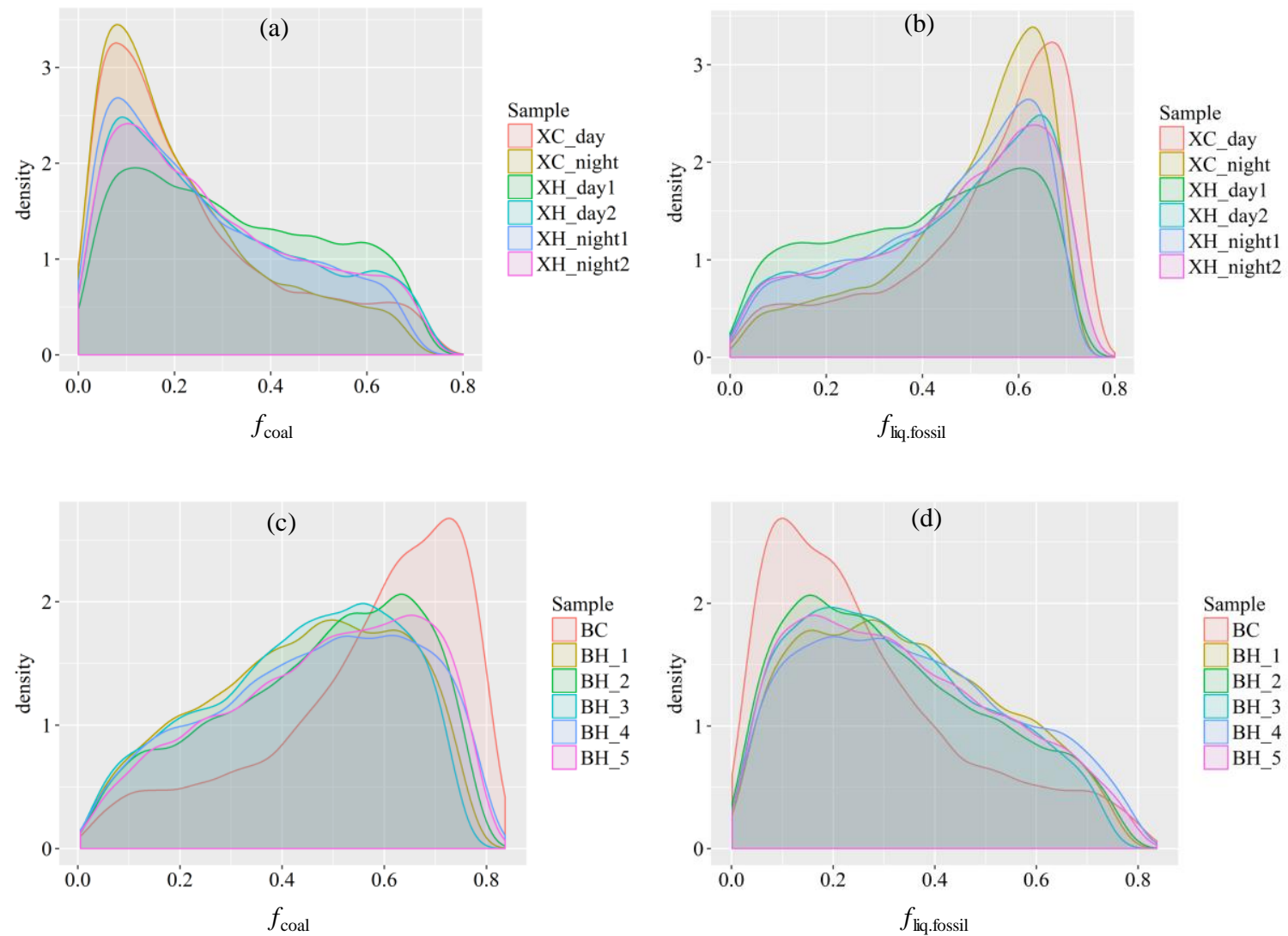

Figure S4. Probability density functions (PDFs) of the relative source contribution of coal combustion $\left(f_{\text {coal }}\right)$ in Xi'an (a) and Beijing (c). PDFs of the relative source contribution of liquid fossil fuel combustion ( $\left.f_{\text {liq.fossil }}\right)$ in Xi'an (b) and Beijing (d). $f_{\text {coal }}$ and $f_{\text {liq.fossil }}$ are constrained by combining radiocarbon and $\delta^{13} \mathrm{C}$ measurements of EC, calculated using the Bayesian Markov chain Monte Carlo approach. For details, see Sect. 2.4. 

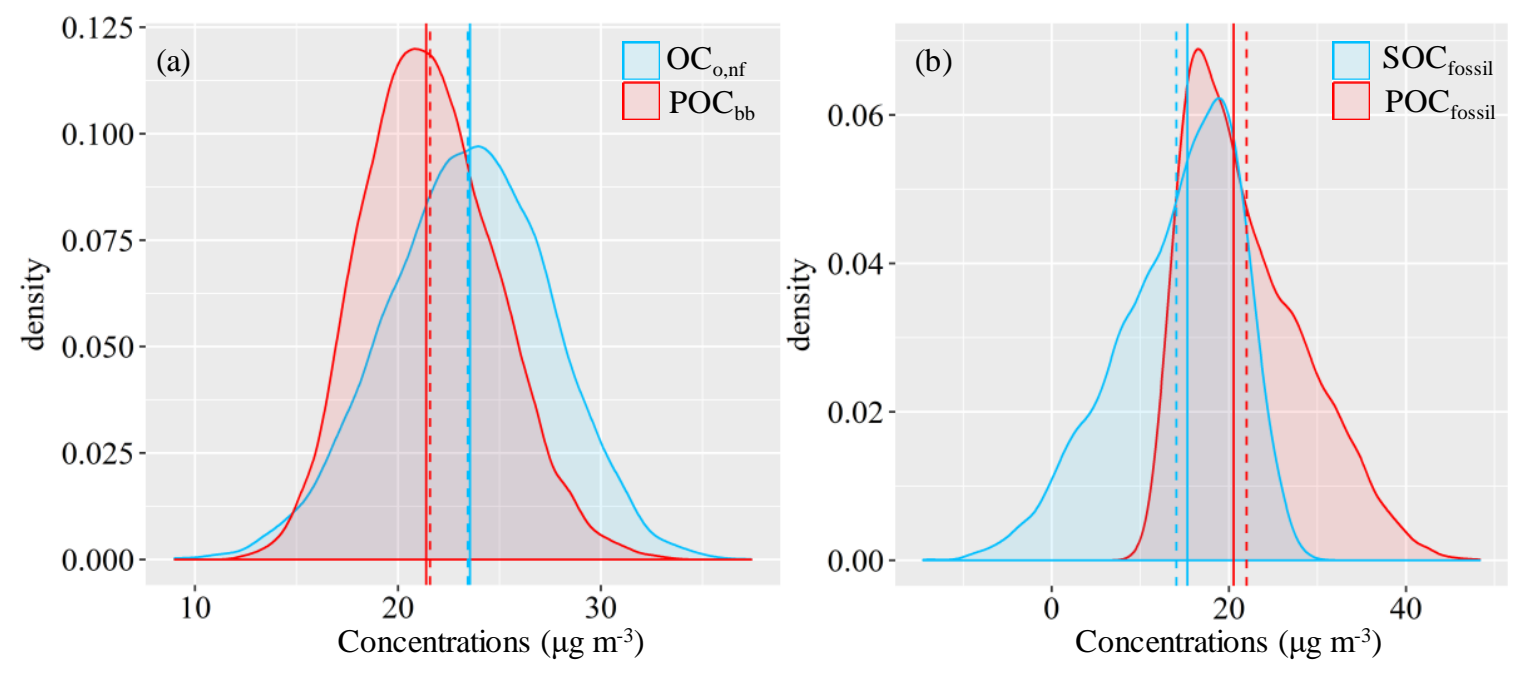

Figure S5. (a) Example probability density functions (PDFs) of concentrations of $\mathrm{OC}_{\mathrm{o}, \mathrm{nf}}$ (light blue) and $\mathrm{POC}_{\mathrm{bb}}$ (red) for sample $\mathrm{XH}_{-}$day2. (b) PDFs of concentrations of $\mathrm{SOC}_{\text {fossil }}$ (light blue) and $\mathrm{POC}_{\text {fossil }}$ (red) for the same sample. Their concentrations are estimated from ${ }^{14} \mathrm{C}$-apportioned $\mathrm{OC}$ and $\mathrm{EC}$ using the EC tracer method (Sect. 2.4). The mean and median are indicated by the dashed and solid vertical lines, respectively. 
Table S1. Details of sampling information and selected samples for radiocarbon measurements.

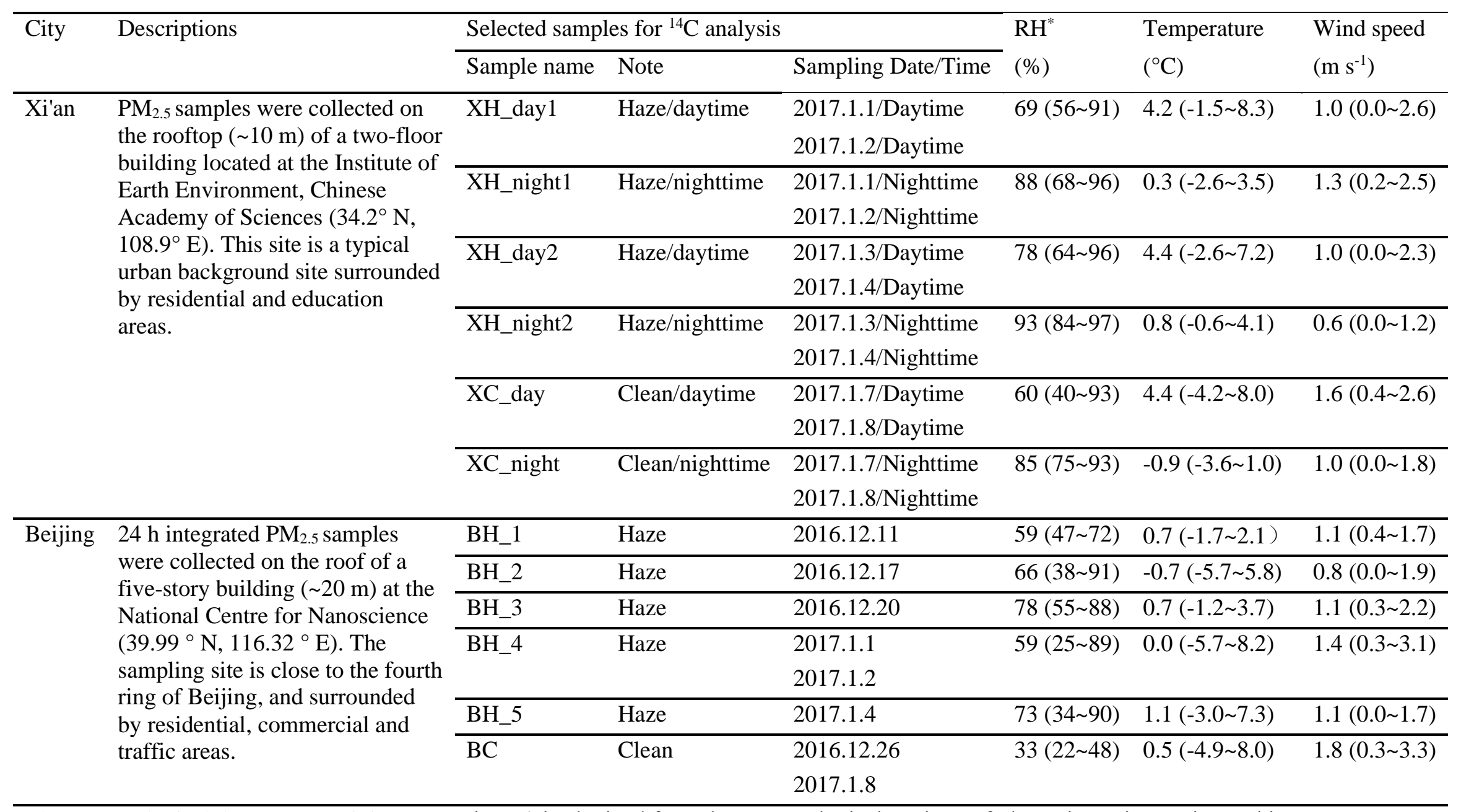

*The meteorological data (mean; minimum-maximum) is obtained from the Meteorological Institute of Shaanxi Province, Xi'an, China. 
Table S2. Fraction modern $\left(\mathrm{F}^{14} \mathrm{C}\right)$ of elemental carbon $(\mathrm{EC})$, organic carbon $(\mathrm{OC})\left(\mathrm{F}^{14} \mathrm{C}_{(\mathrm{EC})}\right.$ and $\mathrm{F}^{14} \mathrm{C}_{(\mathrm{OC})}$, respectively), and stable carbon isotopic compositions $\left(\delta^{13} \mathrm{C}, \% \mathrm{o}\right)$ of $\mathrm{EC}\left(\delta^{13} \mathrm{C}_{\mathrm{EC}}\right)$.

\begin{tabular}{|c|c|c|c|c|c|}
\hline Sample name & Note & Sampling Date/Time & $\mathrm{F}^{14} \mathrm{C}_{(\mathrm{EC})}{ }^{\mathrm{a}}$ & $\mathrm{F}^{14} \mathrm{C}_{(\mathrm{OC})}{ }^{\mathrm{a}}$ & $\delta^{13} \mathrm{C}_{\mathrm{EC}} \mathrm{C}^{\mathrm{a}}$ \\
\hline \multirow[t]{2}{*}{ XH_day1 } & Haze/daytime & 2017.1.1/Daytime & $0.301 \pm 0.007$ & $0.594 \pm 0.004$ & $-24.38 \pm 0.02$ \\
\hline & & 2017.1.2/Daytime & & & \\
\hline \multirow[t]{2}{*}{ XH_night1 } & Haze/nighttime & 2017.1.1/Nighttime & $0.321 \pm 0.014$ & $0.608 \pm 0.005$ & $-25.02 \pm 0.01$ \\
\hline & & 2017.1.2/Nighttime & & & \\
\hline \multirow[t]{2}{*}{ XH_day2 } & Haze/daytime & 2017.1.3/Daytime & $0.287 \pm 0.009$ & $0.605 \pm 0.004$ & $-24.92 \pm 0.04$ \\
\hline & & 2017.1.4/Daytime & & & \\
\hline \multirow[t]{2}{*}{ XH_night2 } & Haze/nighttime & 2017.1.3/Nighttime & $0.288 \pm 0.010$ & $0.603 \pm 0.004$ & $-24.87 \pm 0.03$ \\
\hline & & 2017.1.4/Nighttime & & & \\
\hline \multirow[t]{2}{*}{ XC_day } & Clean/daytime & 2017.1.7/Daytime & $0.273 \pm 0.005$ & $0.550 \pm 0.004$ & $-25.53 \pm 0.02$ \\
\hline & & 2017.1.8/Daytime & & & \\
\hline \multirow[t]{2}{*}{ XC_night } & Clean/nighttime & 2017.1.7/Nighttime & $0.317 \pm 0.004$ & $0.590 \pm 0.004$ & $-25.63 \pm 0.03$ \\
\hline & & 2017.1.8/Nighttime & & & \\
\hline BH_1 & Haze & 2016.12 .11 & $0.243 \pm 0.005$ & $0.383 \pm 0.005$ & $-24.43 \pm 0.03$ \\
\hline BH_2 & Haze & 2016.12 .17 & $0.233 \pm 0.005$ & $0.368 \pm 0.005$ & $-24.09 \pm 0.04$ \\
\hline BH_3 & Haze & 2016.12 .20 & $0.266 \pm 0.005$ & $0.390 \pm 0.004$ & $-24.38 \pm 0.02$ \\
\hline \multirow[t]{2}{*}{ BH_4 } & Haze & 2017.1.1 & $0.203 \pm 0.005$ & $0.333 \pm 0.004$ & $-24.29 \pm 0.02$ \\
\hline & & 2017.1.2 & & & \\
\hline BH_5 & Haze & 2017.1.4 & $0.211 \pm 0.003$ & $0.334 \pm 0.003$ & $-24.15 \pm 0.01$ \\
\hline \multirow[t]{2}{*}{$\mathrm{BC}$} & Clean & 2016.12 .26 & $0.188 \pm 0.006$ & $0.403 \pm 0.005$ & $-23.41 \pm 0.01$ \\
\hline & & 2017.1.8 & & & \\
\hline
\end{tabular}

${ }^{a}$ Values are given in average \pm measurement uncertainty. 
Table S3. Fraction of non-fossil carbon in EC and OC $\left(f_{\mathrm{bb}}(\mathrm{EC}), f_{\mathrm{nf}}(\mathrm{OC})\right)$, fraction of fossil carbon in EC and OC $\left(f_{\text {fossil }}(\mathrm{EC}), f_{\text {fossil }}(\mathrm{OC})\right)$, concentrations $\left(\mu \mathrm{g} \mathrm{m}^{-3}\right)$ of $\mathrm{EC}$ and $\mathrm{OC}$ from non-fossil sources $\left(\mathrm{EC}_{\mathrm{bb}}\right.$ and $\left.\mathrm{OC}_{\mathrm{nf}}\right)$ and fossil sources $\left(\mathrm{EC}_{\text {fossil }}\right.$ and $\left.\mathrm{OC}_{\text {fossil }}\right)$ during haze and clean periods in $\mathrm{Xi}$ 'an and Beijing during December 2016 and January 2017. Details of samples are shown in Table S1.

\begin{tabular}{|l|l|l|l|l|l|l|l|l|}
\hline Sample name & $f_{\mathrm{bb}}(\mathrm{EC})$ & $f_{\text {fossil }}(\mathrm{EC})$ & $f_{\mathrm{nf}}(\mathrm{OC})$ & $f_{\text {fossil }}(\mathrm{OC})$ & $\mathrm{EC}_{\mathrm{bb}}$ & $\mathrm{EC}_{\text {fossil }}$ & $\mathrm{OC}_{\mathrm{nf}}$ \\
\hline XH_day1 & $0.274 \pm 0.008$ & $0.726 \pm 0.008$ & $0.545 \pm 0.011$ & $0.455 \pm 0.011$ & $3.48 \pm 0.36$ & $9.24 \pm 0.91$ & $28.49 \pm 1.53$ & $23.74 \pm 1.31$ \\
\hline XH_night1 & $0.292 \pm 0.014$ & $0.708 \pm 0.014$ & $0.558 \pm 0.011$ & $0.442 \pm 0.011$ & $4.23 \pm 0.47$ & $10.24 \pm 1.05$ & $31.23 \pm 1.70$ & $24.77 \pm 1.40$ \\
\hline XH_day2 & $0.261 \pm 0.010$ & $0.739 \pm 0.010$ & $0.555 \pm 0.011$ & $0.445 \pm 0.011$ & $5.37 \pm 0.58$ & $15.22 \pm 1.55$ & $45.04 \pm 2.41$ & $36.08 \pm 2.02$ \\
\hline XH_night2 & $0.262 \pm 0.010$ & $0.738 \pm 0.010$ & $0.553 \pm 0.011$ & $0.447 \pm 0.011$ & $6.37 \pm 0.69$ & $17.96 \pm 1.82$ & $48.97 \pm 2.62$ & $39.55 \pm 2.22$ \\
\hline XC_day & $0.248 \pm 0.006$ & $0.752 \pm 0.006$ & $0.505 \pm 0.010$ & $0.495 \pm 0.010$ & $2.17 \pm 0.23$ & $6.56 \pm 0.66$ & $12.61 \pm 0.68$ & $12.37 \pm 0.66$ \\
\hline XC_night & $0.288 \pm 0.006$ & $0.712 \pm 0.006$ & $0.542 \pm 0.011$ & $0.458 \pm 0.011$ & $1.76 \pm 0.18$ & $4.33 \pm 0.44$ & $9.31 \pm 0.50$ & $7.88 \pm 0.44$ \\
\hline BH_1 & $0.221 \pm 0.006$ & $0.779 \pm 0.006$ & $0.352 \pm 0.008$ & $0.648 \pm 0.008$ & $3.04 \pm 0.32$ & $10.71 \pm 1.08$ & $15.02 \pm 0.83$ & $27.66 \pm 1.44$ \\
\hline BH_2 & $0.212 \pm 0.006$ & $0.788 \pm 0.006$ & $0.337 \pm 0.008$ & $0.663 \pm 0.008$ & $2.80 \pm 0.29$ & $10.44 \pm 1.04$ & $13.13 \pm 0.71$ & $25.81 \pm 1.31$ \\
\hline BH_3 & $0.242 \pm 0.006$ & $0.758 \pm 0.006$ & $0.358 \pm 0.008$ & $0.642 \pm 0.008$ & $3.97 \pm 0.41$ & $12.42 \pm 1.25$ & $18.62 \pm 1.01$ & $33.43 \pm 1.72$ \\
\hline BH_4 & $0.185 \pm 0.006$ & $0.815 \pm 0.006$ & $0.306 \pm 0.007$ & $0.694 \pm 0.007$ & $2.24 \pm 0.23$ & $9.86 \pm 0.97$ & $11.35 \pm 0.62$ & $25.77 \pm 1.29$ \\
\hline BH_5 & $0.191 \pm 0.004$ & $0.809 \pm 0.004$ & $0.306 \pm 0.007$ & $0.694 \pm 0.007$ & $2.31 \pm 0.24$ & $9.74 \pm 0.97$ & $12.23 \pm 0.67$ & $27.68 \pm 1.42$ \\
\hline BC & $0.171 \pm 0.006$ & $0.829 \pm 0.006$ & $0.370 \pm 0.008$ & $0.630 \pm 0.008$ & $0.27 \pm 0.03$ & $1.33 \pm 0.13$ & $2.54 \pm 0.14$ & $4.34 \pm 0.23$ \\
\hline
\end{tabular}


Table S4. Concentrations $\left(\mu \mathrm{g} \mathrm{m}^{-3}\right)$ of primary $\mathrm{OC}$ from biomass burning $\left(\mathrm{POC}_{\mathrm{bb}}\right)$, primary $\mathrm{OC}$ from fossil sources, $\mathrm{OC}$ from non-fossil sources excluding primary biomass burning $\left(\mathrm{OC}_{\mathrm{o}, \mathrm{nf}}\right)$, secondary $\mathrm{OC}$ from fossil sources $\left(\mathrm{SOC}_{\text {fossil }}\right)$ and total $\mathrm{SOC}$ (i.e., approximately the sum of $\mathrm{OC}_{\mathrm{o}, \mathrm{nf}}$ and SOC $_{\text {fossil }}$ ) during haze and clean periods in Xi'an and Beijing during December 2016 and January 2017 (median and interquartile range). Details of samples are shown in Table S1.

\begin{tabular}{|c|c|c|c|c|c|c|}
\hline Sample name & $\mathrm{POC}_{\mathrm{bb}}$ & $\mathrm{POC}_{\text {fossil }}$ & $\mathrm{OC}_{\mathrm{o}, \mathrm{nf}}$ & $\mathrm{SOC}_{\text {fossil }}$ & SOC & $f_{\text {fossil }}(\mathrm{SOC})$ \\
\hline XH_day 1 & $\begin{array}{l}13.82 \\
(12.50-15.26)\end{array}$ & $\begin{array}{l}13.38 \\
(10.61-16.88)\end{array}$ & $\begin{array}{l}14.58 \\
(12.83-16.25)\end{array}$ & $\begin{array}{l}10.34 \\
(6.71-13.16)\end{array}$ & $\begin{array}{l}24.56 \\
(20.53-28.25)\end{array}$ & $\begin{array}{l}0.41 \\
(0.32-0.47)\end{array}$ \\
\hline XH_night1 & $\begin{array}{l}16.80 \\
(15.14-18.58)\end{array}$ & $\begin{array}{l}13.80 \\
(11.24-17.61)\end{array}$ & $\begin{array}{l}14.40 \\
(12.27-16.41)\end{array}$ & $\begin{array}{l}10.82 \\
(7.04-13.61)\end{array}$ & $\begin{array}{l}24.81 \\
(20.35-28.80)\end{array}$ & $\begin{array}{l}0.43 \\
(0.33-0.49)\end{array}$ \\
\hline XH_day2 & $\begin{array}{l}21.43 \\
(19.28-23.68)\end{array}$ & $\begin{array}{l}20.50 \\
(16.60-26.58)\end{array}$ & $\begin{array}{l}23.54 \\
(20.75-26.20)\end{array}$ & $\begin{array}{l}15.41 \\
(9.35-19.52)\end{array}$ & $\begin{array}{l}38.29 \\
(31.42-44.04)\end{array}$ & $\begin{array}{l}0.40 \\
(0.29-0.45)\end{array}$ \\
\hline XH_night2 & $\begin{array}{l}25.40 \\
(22.88-28.07)\end{array}$ & $\begin{array}{l}24.32 \\
(19.66-30.96)\end{array}$ & $\begin{array}{l}23.51 \\
(20.34-26.62)\end{array}$ & $\begin{array}{l}15.06 \\
(8.37-19.94)\end{array}$ & $\begin{array}{l}37.85 \\
(30.55-44.63)\end{array}$ & $\begin{array}{l}0.39 \\
(0.27-0.46)\end{array}$ \\
\hline XC_day & $\begin{array}{l}8.64 \\
(7.77-9.54)\end{array}$ & $\begin{array}{l}8.21 \\
(6.82-10.63)\end{array}$ & $\begin{array}{l}3.98 \\
(2.95-4.94)\end{array}$ & $\begin{array}{l}4.07 \\
(1.73-5.59)\end{array}$ & $\begin{array}{l}7.76 \\
(5.18-9.89)\end{array}$ & $\begin{array}{l}0.52 \\
(0.36-0.60)\end{array}$ \\
\hline XC_night & $\begin{array}{l}6.99 \\
(6.30-7.72)\end{array}$ & $\begin{array}{l}5.37 \\
4.46-6.85\end{array}$ & $\begin{array}{l}2.32 \\
1.51-3.07\end{array}$ & $\begin{array}{l}2.47 \\
1.00-3.43\end{array}$ & $\begin{array}{l}4.57 \\
2.83-6.07\end{array}$ & $\begin{array}{l}0.53 \\
0.38-0.64\end{array}$ \\
\hline BH_1 & $\begin{array}{l}12.15 \\
(10.90-13.44)\end{array}$ & $\begin{array}{l}18.32 \\
(14.82-21.65)\end{array}$ & $\begin{array}{l}2.87 \\
(1.47-4.27)\end{array}$ & $\begin{array}{l}9.44 \\
(5.86-12.99)\end{array}$ & $\begin{array}{l}12.33 \\
98.13-16.41)\end{array}$ & $\begin{array}{l}0.76 \\
(0.67-0.86)\end{array}$ \\
\hline BH_2 & $\begin{array}{l}11.17 \\
(10.04-12.34)\end{array}$ & $\begin{array}{l}18.36 \\
(14.89-21.44)\end{array}$ & $\begin{array}{l}1.98 \\
(0.65-3.17)\end{array}$ & $\begin{array}{l}7.56 \\
(4.26-11.04)\end{array}$ & $\begin{array}{l}9.59 \\
(5.67-13.45)\end{array}$ & $\begin{array}{l}0.80 \\
(0.68-0.91)\end{array}$ \\
\hline BH_3 & $\begin{array}{l}15.82 \\
(14.21-17.56)\end{array}$ & $\begin{array}{l}21.21 \\
(17.18-25.02)\end{array}$ & $\begin{array}{l}2.77 \\
(0.95-4.50)\end{array}$ & $\begin{array}{l}12.24 \\
(8.16-16.36)\end{array}$ & $\begin{array}{l}15.01 \\
(10.19-19.75)\end{array}$ & $\begin{array}{l}0.81 \\
(0.72-0.92)\end{array}$ \\
\hline BH_4 & $\begin{array}{l}8.88 \\
(8.00-9.84)\end{array}$ & $\begin{array}{l}16.95 \\
(13.83-19.94)\end{array}$ & $\begin{array}{l}2.45 \\
(1.41-3.44)\end{array}$ & $\begin{array}{l}8.79 \\
(5.70-12.06)\end{array}$ & $\begin{array}{l}11.30 \\
(7.71-14.83)\end{array}$ & $\begin{array}{l}0.78 \\
(0.70-0.86)\end{array}$ \\
\hline BH_5 & $\begin{array}{l}9.17 \\
(8.25-10.17)\end{array}$ & $\begin{array}{l}16.94 \\
(13.73-19.86)\end{array}$ & $\begin{array}{l}3.00 \\
(1.96-4.07)\end{array}$ & $\begin{array}{l}10.79 \\
(7.65-14.08)\end{array}$ & $\begin{array}{l}13.82 \\
(10.26-17.53)\end{array}$ & $\begin{array}{l}0.78 \\
(0.71-0.85)\end{array}$ \\
\hline $\mathrm{BC}$ & $\begin{array}{l}1.09 \\
(0.98-1.21)\end{array}$ & $\begin{array}{l}.55 \\
(2.15-2.90)\end{array}$ & $\begin{array}{l}1.44 \\
(1.30-1.59)\end{array}$ & $\begin{array}{l}1.80 \\
(1.42-2.22)\end{array}$ & $\begin{array}{l}3.26 \\
(2.79-3.76)\end{array}$ & $\begin{array}{l}0.55 \\
(0.50-0.60)\end{array}$ \\
\hline
\end{tabular}


Table S5. Fractional contribution of different incomplete combustion sources to EC in different seasons $\left(f_{\mathrm{bb}}, f_{\text {liq.fossil }}\right.$ and $f_{\text {coal }}$; median and interquartile range ), and $\mathrm{EC}$ concentrations $\left(\mu \mathrm{g} \mathrm{m}^{-3}\right)$ from biomass burning $\left(\mathrm{EC}_{\mathrm{bb}}\right)$, coal combustion $\left(\mathrm{EC}_{\mathrm{coal}}\right)$ and liquid fossil fuel combustion $\left(\mathrm{EC}_{\text {liq.fossil }}\right)$ (median and interquartile range). Details of samples are shown in Table S1.

\begin{tabular}{|c|c|c|c|c|c|c|c|c|}
\hline City & Sample Name & Note & $f_{\mathrm{bb}}$ & $f_{\text {liq.fossil }}$ & $f_{\text {coal }}$ & $\mathrm{EC}_{\mathrm{bb}}$ & $\mathrm{EC}_{\text {liq.fossil }}$ & $\mathrm{EC}_{\text {coal }}$ \\
\hline \multirow[t]{6}{*}{ Xi'an } & XH_day1 & Haze1/daytime & $\begin{array}{l}0.27 \\
(0.27-0.28)\end{array}$ & $\begin{array}{l}0.44 \\
(0.26-0.58)\end{array}$ & $\begin{array}{l}0.29 \\
(0.14-0.47)\end{array}$ & $\begin{array}{l}3.48 \\
(3.24-3.73)\end{array}$ & $\begin{array}{l}5.49 \\
(3.10-7.30)\end{array}$ & $\begin{array}{l}.66 \\
(1.82-5.98)\end{array}$ \\
\hline & XH_night1 & Haze1/nighttime & $\begin{array}{l}0.29 \\
(0.29-0.30)\end{array}$ & $\begin{array}{l}0.47 \\
(0.30-0.59)\end{array}$ & $\begin{array}{l}0.23 \\
(0.12-0.41)\end{array}$ & $\begin{array}{l}4.23 \\
(3.93-4.54)\end{array}$ & $\begin{array}{l}6.75 \\
(4.28-8.45)\end{array}$ & $\begin{array}{l}3.29 \\
(1.69-5.87)\end{array}$ \\
\hline & XH_day2 & Haze2/daytime & $\begin{array}{l}0.29 \\
(0.29-0.30)\end{array}$ & $\begin{array}{l}0.47 \\
(0.30-0.59)\end{array}$ & $\begin{array}{l}0.23 \\
(0.12-0.41)\end{array}$ & $\begin{array}{l}5.38 \\
(4.99-5.78)\end{array}$ & $\begin{array}{l}10.04 \\
(6.15-12.60)\end{array}$ & $\begin{array}{l}.94 \\
(2.44-8.94)\end{array}$ \\
\hline & XH_night2 & Haze2/nighttime & $\begin{array}{l}0.26 \\
(0.26-0.27)\end{array}$ & $\begin{array}{l}0.49 \\
(0.30-0.62)\end{array}$ & $\begin{array}{l}0.25 \\
(0.12-0.44)\end{array}$ & $\begin{array}{l}6.39 \\
(5.94-6.85)\end{array}$ & $\begin{array}{l}11.85 \\
(7.40-14.93)\end{array}$ & $\begin{array}{l}5.88 \\
(2.91-10.35)\end{array}$ \\
\hline & XC_day & Clean/daytime & $\begin{array}{l}0.25 \\
(0.24-0.26)\end{array}$ & $\begin{array}{l}0.57 \\
(0.40-0.66)\end{array}$ & $\begin{array}{l}0.18 \\
(0.09-0.35)\end{array}$ & $\begin{array}{l}2.17 \\
(2.01-2.32)\end{array}$ & $\begin{array}{l}4.73 \\
(3.23-5.68)\end{array}$ & $\begin{array}{l}1.69 \\
(0.83-3.24)\end{array}$ \\
\hline & XC_night & Clean/nighttime & $\begin{array}{l}0.29 \\
(0.28-0.30)\end{array}$ & $\begin{array}{l}0.54 \\
(0.38-0.62)\end{array}$ & $\begin{array}{l}0.17 \\
(0.09-0.33)\end{array}$ & $\begin{array}{l}1.76 \\
(1.63-1.89)\end{array}$ & $\begin{array}{l}3.17 \\
(2.21-3.76)\end{array}$ & $\begin{array}{l}1.07 \\
(0.53-2.05)\end{array}$ \\
\hline \multirow[t]{6}{*}{ Beijing } & BH_1 & Haze1 & $\begin{array}{l}0.22 \\
(0.21-0.23)\end{array}$ & $\begin{array}{l}0.32 \\
(0.18-0.49)\end{array}$ & $\begin{array}{l}0.46 \\
(0.29-0.60)\end{array}$ & $\begin{array}{l}3.05 \\
(2.83-3.28)\end{array}$ & $\begin{array}{l}4.38 \\
(2.49-6.68)\end{array}$ & $\begin{array}{l}6.18 \\
(3.90-8.18)\end{array}$ \\
\hline & BH_2 & Haze2 & $\begin{array}{l}0.21 \\
(0.21-0.22)\end{array}$ & $\begin{array}{l}0.29 \\
(0.16-0.46)\end{array}$ & $\begin{array}{l}0.50 \\
(0.33-0.62)\end{array}$ & $\begin{array}{l}2.80 \\
(2.59-3.02)\end{array}$ & $\begin{array}{l}3.89 \\
(2.15-6.13)\end{array}$ & $\begin{array}{l}6.42 \\
(4.20-8.15)\end{array}$ \\
\hline & BH_3 & Haze3 & $\begin{array}{l}0.24 \\
(0.23-0.25)\end{array}$ & $\begin{array}{l}0.31 \\
(0.18-0.47)\end{array}$ & $\begin{array}{l}0.45 \\
(0.29-0.58)\end{array}$ & $\begin{array}{l}3.98 \\
(3.69-4.28)\end{array}$ & $\begin{array}{l}5.14 \\
(2.99-7.79)\end{array}$ & $\begin{array}{l}7.17 \\
(4.52-9.38)\end{array}$ \\
\hline & BH_4 & Haze4 & $\begin{array}{l}0.19 \\
(0.18-0.19)\end{array}$ & $\begin{array}{l}0.33 \\
(0.18-0.50)\end{array}$ & $\begin{array}{l}0.49 \\
(0.31-0.63)\end{array}$ & $\begin{array}{l}2.24 \\
(2.07-2.40)\end{array}$ & $\begin{array}{l}3.90 \\
(2.21-6.00)\end{array}$ & $\begin{array}{l}5.80 \\
(3.75-7.53)\end{array}$ \\
\hline & BH_5 & Haze5 & \begin{tabular}{|l|}
0.19 \\
$(0.19-0.20)$
\end{tabular} & $\begin{array}{l}0.31 \\
(0.17-0.48)\end{array}$ & $\begin{array}{l}0.50 \\
(0.33-0.63)\end{array}$ & $\begin{array}{l}2.31 \\
(2.13-2.48)\end{array}$ & $\begin{array}{l}3.75 \\
(2.09-5.87)\end{array}$ & $\begin{array}{l}5.86 \\
(3.73-7.55)\end{array}$ \\
\hline & $\mathrm{BC}$ & Clean & $\begin{array}{l}0.17 \\
(0.17-0.18)\end{array}$ & $\begin{array}{l}0.22 \\
(0.12-0.37)\end{array}$ & $\begin{array}{l}0.61 \\
(0.46-0.71)\end{array}$ & $\begin{array}{l}0.27 \\
(0.25-0.30)\end{array}$ & $\begin{array}{l}0.35 \\
(0.18-0.59)\end{array}$ & $\begin{array}{l}0.96 \\
(0.72-1.14)\end{array}$ \\
\hline
\end{tabular}


Table S6. Fractional contribution of different incomplete combustion sources to EC (median and interquartile range).

\begin{tabular}{|ll|l|l|l|}
\hline & & Biomass burning & Coal combustion & $\begin{array}{l}\text { Liquid fossil fuel combustion } \\
\text { (i.e., vehicle emissions) }\end{array}$ \\
\hline Xi'an haze day & $\begin{array}{l}0.27 \\
(0.26-0.27)\end{array}$ & $\begin{array}{l}0.26 \\
(0.13-0.44)\end{array}$ & $\begin{array}{l}0.47 \\
(0.29-0.60)\end{array}$ \\
\hline Xi'an haze night & $\begin{array}{l}0.28 \\
(0.27-0.28)\end{array}$ & $\begin{array}{l}0.23 \\
(0.12-0.41)\end{array}$ & $\begin{array}{l}0.49 \\
(0.31-0.61)\end{array}$ \\
\hline Xi'an clean day & $\begin{array}{l}0.25 \\
(0.24-0.26)\end{array}$ & $\begin{array}{l}0.18 \\
(0.09-0.33)\end{array}$ & $\begin{array}{l}0.57 \\
(0.42-0.66)\end{array}$ \\
\hline Xi'an clean night & 0.29 & 0.18 & 0.53 \\
& & $(0.28-0.30)$ & $(0.09-0.34)$ & $(0.37-0.62)$ \\
\hline Beijing haze & 0.21 & 0.48 & 0.31 \\
& & $(0.20-0.22)$ & $(0.31-0.61)$ & $(0.18-0.48)$ \\
\hline Beijing clean & 0.17 & 0.61 & 0.22 \\
& & $(0.17-0.18)$ & $(0.45-0.71)$ & $(0.12-0.38)$ \\
\hline
\end{tabular}




\section{References}

Dusek, U., Monaco, M., Prokopiou, M., Gongriep, F., Hitzenberger, R., Meijer, H. A. J., and Röckmann, T.: Evaluation of a two-step thermal method for separating organic and elemental carbon for radiocarbon analysis, Atmos. Meas. Tech., 7, 1943-1955, https://doi.org/10.5194/amt7-1943-2014, 2014.

Ni, H., Huang, R.-J., Cao, J., Liu, W., Zhang, T., Wang, M., Meijer, H. A. J., and Dusek, U.: Source apportionment of carbonaceous aerosols in Xi'an, China: insights from a full year of measurements of radiocarbon and the stable isotope ${ }^{13} \mathrm{C}$, Atmos. Chem. Phys., 18, 16363-16383, https://doi.org/10.5194/acp-18-16363-2018, 2018.

Zenker, K., Vonwiller, M., Szidat, S., Calzolai, G., Giannoni, M., Bernardoni, V., Jedynska, A. D., Henzing, B., Meijer, H. A., and Dusek, U.: Evaluation and Inter-comparison of oxygen-based OCEC separation methods for radiocarbon analysis of ambient aerosol particle samples, Atmosphere, 8, 226, https://doi.org/10.3390/atmos8110226, 2017.

Zhang, Y. L., Li, J., Zhang, G., Zotter, P., Huang, R.-J., Tang, J.-H., Wacker, L., Prévôt, A. S. H., and Szidat, S.: Radiocarbon-based source apportionment of carbonaceous aerosols at a regional background site on Hainan Island, South China, Environ. Sci. Technol., 48, 26512659, https://doi.org/10.1021/es4050852, 2014.

Zhang, Y. L., Huang, R. J., El Haddad, I., Ho, K. F., Cao, J. J., Han, Y., Zotter, P., Bozzetti, C., Daellenbach, K. R., Canonaco, F., Slowik, J. G., Salazar, G., Schwikowski, M., Schnelle-Kreis, J., Abbaszade, G., Zimmermann, R., Baltensperger, U., Prévôt, A. S. H., and Szidat, S.: Fossil vs. nonfossil sources of fine carbonaceous aerosols in four Chinese cities during the extreme winter haze episode of 2013, Atmos. Chem. Phys., 15, 1299-1312, https://doi.org/10.5194/acp-15-1299-2015, 2015 . 